\title{
Discrete Neumann boundary value problem for a nonlinear equation with singular $\phi$-Laplacian
}

Man Xu and Ruyun Ma*

\section{"Correspondence:}

mary@nwnu.edu.cn

Department of Mathematics,

Northwest Normal University,

Lanzhou, 730070, P.R. China

\section{Springer}
Abstract
Let $I \subset \mathbb{R}$ be an open interval with $0 \in I$, and let $g \in C^{1}(I,(0,+\infty))$. Let $N \in \mathbb{N}$ be an integer with $N \geq 4,[2, N-1]_{\mathbb{Z}}:=\{2,3, \ldots, N-1\}$. We are concerned with the existence of solutions for the discrete Neumann problem

$$
\left\{\begin{array}{l}
\nabla\left(k^{n-1} \frac{\Delta v_{k}}{\sqrt{1-\left(\Delta v_{k}\right)^{2}}}\right)=n k^{n-1}\left[-\frac{g^{\prime}\left(\psi^{-1}\left(v_{k}\right)\right)}{\sqrt{1-\left(\Delta v_{k}\right)^{2}}}+g\left(\psi^{-1}\left(v_{k}\right)\right) H\left(\psi^{-1}\left(v_{k}\right), k\right)\right], \quad k \in[2, N-1]_{\mathbb{Z}} \\
\Delta v_{1}=0=\Delta v_{N-1}
\end{array}\right.
$$

which is a discrete analogue of the Neumann problem about the rotationally symmetric spacelike graphs with a prescribed mean curvature function in some Friedmann-Lemaitre-Robertson-Walker (FLRW) spacetimes, where $\psi(s):=\int_{0}^{s} \frac{d t}{g(t)}, \psi^{-1}$ is the inverse function of $\psi$, and $H: \mathbb{R} \times[2, N-1]_{\mathbb{Z}} \rightarrow \mathbb{R}$ is continuous with respect to the first variable. The proofs of the main results are based upon the Brouwer degree theory.

MSC: 34B10; 34B18; 39A11; 47H11

Keywords: prescribed mean curvature function; singular $\phi$-Laplacian; existence; Friedmann-Lemaître-Robertson-Walker spacetime; Neumann problem; Brouwer degree

\section{Introduction}

Up to the last decade, little attention has been paid to the graphs of Dirichlet or Neumann boundary value problems for the prescribed mean curvature equation in some Friedmann-Lemaître-Robertson-Walker (FLRW) spacetimes; see [1, 2]. Recently, Mawhin and Torres [1] studied the existence of radially symmetric solutions for the Neumann problem with a prescribed mean curvature function in a certain family of FLRW spacetimes

$$
\left\{\begin{array}{l}
\operatorname{div}\left(\frac{\operatorname{grad} u}{g(u) \sqrt{g(u)^{2}-|\operatorname{grad} u|^{2}}}\right)+\frac{g^{\prime}(u)}{\sqrt{g(u)^{2}-|\operatorname{grad} u|^{2}}}\left(n+\frac{|\operatorname{grad} u|^{2}}{g(u)^{2}}\right)=n H(u,|x|), \\
|\operatorname{grad} u|<g(u) \quad \text { in } B(R), \\
\frac{\partial u}{\partial \nu}=0 \quad \text { in } \partial B(R),
\end{array}\right.
$$

(0) The Author(s) 2018. This article is distributed under the terms of the Creative Commons Attribution 4.0 International License (http://creativecommons.org/licenses/by/4.0/), which permits unrestricted use, distribution, and reproduction in any medium, provided you give appropriate credit to the original author(s) and the source, provide a link to the Creative Commons license, and indicate if changes were made. 
which, as it is well known, plays an important role in cosmology, where $B(R)=\left\{x \in \mathbb{R}^{n}\right.$ : $|x|<R\}, \frac{\partial u}{\partial v}$ denotes the outward normal derivative of $u, H: \mathbb{R} \times[0,+\infty) \rightarrow \mathbb{R}$ is the prescribed mean curvature function, $g \in C^{1}(\mathbb{R})$ is the radius of the Universe at time $t$, and $\frac{g^{\prime}(t)}{g(t)}$ is the Hubble's rate. By using the radial coordinate change, (1.1) can be reduced to a Neumann problem of quasilinear ordinary differential equation; see (6) in [1]. Its discrete analogue is the following:

$$
\left\{\begin{array}{l}
\nabla\left(k^{n-1} \phi\left(\Delta v_{k}\right)\right)=n k^{n-1}\left[-\frac{g^{\prime}\left(\psi^{-1}\left(v_{k}\right)\right)}{\sqrt{1-\left(\Delta v_{k}\right)^{2}}}+g\left(\psi^{-1}\left(v_{k}\right)\right) H\left(\psi^{-1}\left(v_{k}\right), k\right)\right] \\
k \in[2, N-1]_{\mathbb{Z}} \\
\Delta v_{1}=0=\Delta v_{N-1}
\end{array}\right.
$$

where $\phi:(-1,1) \rightarrow \mathbb{R}$ is an increasing homeomorphism defined by $\phi(s)=\frac{s}{\sqrt{1-s^{2}}}, s \in \mathbb{R}$ (notice that $\phi(0)=0$ ), $\nabla$ is the backward difference operator defined by $\nabla v_{k}=v_{k}-v_{k-1}, \Delta$ is the forward difference operator defined by $\Delta v_{k}=v_{k+1}-v_{k}, g \in C^{1}(I,(0,+\infty)), I \subset \mathbb{R}$ is an open interval with $0 \in I, \psi(s):=\int_{0}^{s} \frac{d t}{g(t)}, \psi^{-1}$ is the inverse function of $\psi, H: \mathbb{R} \times[2, N-$ $1]_{\mathbb{Z}} \rightarrow \mathbb{R}$ is continuous with respect to the first variable, and $[2, N-1]_{\mathbb{Z}}:=\{2,3, \ldots, N-1\}$ with integer $N \geq 4$.

A particular significance in (1.2) lies in the fact that its numerical solutions can be used to guide the numerical computation work. On the other hand, the problem is interesting in itself. For example, when we discretize a differential equation, the properties of its solutions such as the existence, multiplicity, and uniqueness may not be shared between the continuous differential equation and its related discrete difference equation [3, p. 520]. Thus, we have to face new challenges and innovation.

Let $\theta, \eta \in \mathbb{R}$ with $\theta<0<\eta$. Denote $I=(\theta, \eta)$ and $\widehat{I}=[\theta, \eta]$. We always make the following assumptions:

(A1) $g \in C^{1}(I)$ and $g(t)>0$ on $I$;

(A2) $\lim _{t \rightarrow \theta^{+}} \frac{g^{\prime}(t)}{g(t)}=+\infty$ and $\lim _{t \rightarrow \eta^{-}} \frac{g^{\prime}(t)}{g(t)}=-\infty$.

The function $\psi: I \rightarrow \mathbb{R}$ is important in the sequel, therefore, and we rewrite it for the reader's convenience:

$$
\psi(s):=\int_{0}^{s} \frac{d t}{g(t)}
$$

It is obvious that $\psi(0)=0$ and $\psi$ is strictly increasing by (A1).

Let us state the main results of this paper.

Theorem 1.1 Assume that $g$ satisfies (A1) and (A2). Suppose that

$$
\lim _{s \rightarrow \theta^{+}} \psi(s)=-\infty, \quad \lim _{s \rightarrow \eta^{-}} \psi(s)=+\infty,
$$

and

$$
\beta:=\max _{t \in \widehat{I}}\left|g^{\prime}(t)\right|<+\infty
$$


If

$$
N<\frac{1}{\beta}+1
$$

then (1.2) has at least one solution $\mathbf{v}$ for any $H: \widehat{I} \times[2, N-1]_{\mathbb{Z}} \rightarrow \mathbb{R}$.

Theorem 1.2 Assume that $g$ satisfies (A1) and (A2). Then there exists $N_{H}>0$ such that (1.2) has at least one solution $\mathbf{v}$ for any $H: \widehat{I} \times[0,+\infty) \rightarrow \mathbb{R}$ if $N<N_{H}$.

Remark 1.1 It is obvious that the difference between Theorems 1.1 and 1.2 is that the constant $N_{H}$ may depend on the function $H$ in Theorem 1.2, whereas it is uniform for all $H$ in Theorem 1.1.

Remark 1.2 The function $\psi(s)$ in Theorem 1.1 related to the function $g$ has infinite limits at the end points of $I$. However, in some cosmological models, the limits are finite. Inspired by this, we weaken conditions (1.4) and (1.5) and give Theorem 1.2.

It is worth pointing out that the properties of solutions for the prescribed mean curvature problems in the Minkowski space $\mathbb{L}^{n+1}$, which is the case of (1.1) with $g(t) \equiv 1$, have been extensively studied. In this setting, we mention the papers [4-14]. However, in contrast with the continuous results mentioned, the number of references of the corresponding discrete results is significantly lower; see [15-18].

The existence of solutions of the Neumann and periodic boundary value problems of semilinear differential equations has been extensively studied by many authors via the following Mawhin continuation theorem (see [19-23] and references therein).

Lemma A (Mawhin et al. [24, 25]) Let $X$ and $Y$ be two Banach spaces, and let $L: D(L) \subset$ $X \rightarrow Y$ be a Fredholm operator with index zero. Furthermore, let $\Omega \subset X$ be an open bounded set, and let $N: \bar{\Omega} \rightarrow Y$ be L-compact on $\bar{\Omega}$. Suppose that

(1) $L x \neq \lambda N x, x \in \partial \Omega, \lambda \in(0,1)$;

(2) $N x \notin \operatorname{Im} L, x \in \partial \Omega \cap \operatorname{ker} L$; and

(3) the Brouwer degree

$$
\operatorname{deg}(Q N, \Omega \cap \operatorname{ker} L, 0) \neq 0
$$

Then the equation $L x=N x$ has a solution $x \in \bar{\Omega}$.

However, this tool cannot be directly used to deal with the quasilinear problem (1.2). To prove Theorems 1.1 and 1.2, we have to construct an equivalent fixed point problem for (1.2); see Proposition 2.2. This is motivated by Mawhin and Torres [1] to treat the Neumann problems of the quasilinear differential equation (1.1).

For other results on the problems in some FLRW spacetimes, see [26-29] and the references therein.

The rest of the paper is arranged as follows. In Section 2, we give some notations and state some preliminary results. Section 3 is devoted to proving the Theorems 1.1 and 1.2. Finally, we give some examples to illustrate our main results. 


\section{Some notations and preliminary results}

Let us start with some notations. For $\mathbf{v} \in \mathbb{R}^{p}$, let $\|\mathbf{v}\|_{\infty}=\max _{1 \leq k \leq p}\left|v_{k}\right|$. We define $\sum_{k=i}^{j} v_{k}=$ 0 for $j<i$.

Let

$$
W^{N-2}=\left\{\mathbf{v} \in \mathbb{R}^{N}: \Delta v_{1}=0=\Delta v_{N-1}\right\}
$$

with the norm $\|\mathbf{v}\|_{\infty}:=\max _{2 \leq k \leq N-1}\left|v_{k}\right|$.

For any $\mathbf{v}=\left(v_{1}, \ldots, v_{N}\right) \in \mathbb{R}^{N}$, we define

$$
\triangle \mathbf{v}=\left(\Delta v_{1}, \ldots, \Delta v_{N-1}\right) \in \mathbb{R}^{N-1}
$$

as follows:

$$
\Delta v_{k}=v_{k+1}-v_{k}, \quad k \in[1, N-1]_{\mathbb{Z}} .
$$

Further, if $\|\Delta \mathbf{v}\|_{\infty}:=\max _{k \in[1, N-1]_{\mathbb{Z}}}\left|\Delta v_{k}\right|<1$, then we define

$$
\nabla\left(k^{n-1} \phi(\Delta \mathbf{v})\right)=\left(\nabla\left(2^{n-1} \phi\left(\Delta v_{2}\right)\right), \ldots, \nabla\left((N-1)^{n-1} \phi\left(\Delta v_{N-1}\right)\right)\right) \in \mathbb{R}^{N-2}
$$

as follows:

$$
\nabla\left(k^{n-1} \phi\left(\Delta v_{k}\right)\right)=k^{n-1} \phi\left(\Delta v_{k}\right)-(k-1)^{n-1} \phi\left(\Delta v_{k-1}\right), \quad k \in[2, N-1]_{\mathbb{Z}} .
$$

We are first concerned with the following discrete Neumann problem with singular discrete $\phi$-Laplacian:

$$
\left\{\begin{array}{l}
\nabla\left(k^{n-1} \phi\left(\Delta v_{k}\right)\right)=n k^{n-1} h(k), \quad k \in[2, N-1]_{\mathbb{Z}}, \\
\Delta v_{1}=0=\Delta v_{N-1},
\end{array}\right.
$$

where $h:[2, N-1]_{\mathbb{Z}} \rightarrow \mathbb{R}$ satisfies

$$
\sum_{k=2}^{N-1} n k^{n-1} h(k)=0
$$

Proposition 2.1 The Neumann problem (2.1) is solvable if and only if (2.2) is valid, and the form of the solutions of $(2.1)$ is $\left(v_{2}, v_{2}, \ldots, v_{N-1}, v_{N-1}\right)$, where

$$
v_{k}=v_{2}+\sum_{j=2}^{k-1} \phi^{-1}\left(\frac{1}{j^{n-1}} \sum_{i=2}^{j} n i^{n-1} h(i)\right), \quad v_{2} \in \mathbb{R}, k \in[3, N-1]_{\mathbb{Z}} .
$$

Proof By direct computation it is easy to see that

$$
\phi\left(\Delta v_{k}\right)=\frac{1}{k^{n-1}} \sum_{i=2}^{k} n i^{n-1} h(i) .
$$

This fact, together with the boundary conditions, implies (2.2) and (2.3). 
Now, we consider the Neumann problem (1.2). Define $\mathcal{A}: W^{N-2} \rightarrow W^{N-2}$ by $\mathcal{A}(\mathbf{v})=\mathbf{y}$, where

$$
\begin{aligned}
y_{2}= & v_{2}+\frac{1}{N-2} \sum_{k=2}^{N-1} n k^{n-1}\left[-\frac{g^{\prime}\left(\psi^{-1}\left(v_{k}\right)\right)}{\sqrt{1-\left(\triangle v_{k}\right)^{2}}}+g\left(\psi^{-1}\left(v_{k}\right)\right) H\left(\psi^{-1}\left(v_{k}\right), k\right)\right], \\
y_{k}= & v_{2}+\frac{1}{N-2} \sum_{k=2}^{N-1} n k^{n-1}\left[-\frac{g^{\prime}\left(\psi^{-1}\left(v_{k}\right)\right)}{\sqrt{1-\left(\triangle v_{k}\right)^{2}}}+g\left(\psi^{-1}\left(v_{k}\right)\right) H\left(\psi^{-1}\left(v_{k}\right), k\right)\right] \\
& +\sum_{j=2}^{k-1} \phi^{-1}\left(\frac{1}{j^{n-1}} \sum_{i=2}^{j} n i^{n-1}\left[-\frac{g^{\prime}\left(\psi^{-1}\left(v_{i}\right)\right)}{\sqrt{1-\left(\triangle v_{i}\right)^{2}}}+g\left(\psi^{-1}\left(v_{i}\right)\right) H\left(\psi^{-1}\left(v_{i}\right), i\right)\right]\right), \\
k & \in[3, N-1]_{\mathbb{Z}},
\end{aligned}
$$

where $\phi^{-1}$ is the inverse function of $\phi(s)$, namely

$$
\phi^{-1}(s)=\frac{s}{\sqrt{1+s^{2}}}, \quad s \in \mathbb{R} .
$$

Therefore, we get the following fixed point reformulation for (1.2).

Proposition 2.2 $\mathbf{v} \in \mathbb{R}^{N}$ is a solution of (1.2) if and only if $\mathbf{v} \in W^{N-2}$ and $\mathcal{A}(\mathbf{v})=\mathbf{v}$.

Proof Denote

$$
G[v](k)=\sum_{i=2}^{k} n i^{n-1}\left[-\frac{g^{\prime}\left(\psi^{-1}\left(v_{i}\right)\right)}{\sqrt{1-\left(\Delta v_{i}\right)^{2}}}+g\left(\psi^{-1}\left(v_{i}\right)\right) H\left(\psi^{-1}\left(v_{i}\right), i\right)\right] .
$$

With this notation, the function $\mathcal{A}$ is simply written as

$$
\begin{aligned}
& y_{2}=v_{2}+\frac{1}{N-2} G[v](N-1), \\
& y_{k}=v_{2}+\frac{1}{N-2} G[v](N-1)+\sum_{j=2}^{k-1} \phi^{-1}\left(\frac{1}{j^{n-1}} G[v](j)\right), \quad k \in[3, N-1]_{\mathbb{Z}} .
\end{aligned}
$$

If $\mathbf{v}=\mathcal{A}(\mathbf{v})$, then, taking $k=2$, we have

$$
v_{2}=v_{2}+\frac{1}{N-2} G[v](N-1)
$$

that is,

$$
G[v](N-1)=0 .
$$

On the other hand, for any $k \in[2, N-1]_{\mathbb{Z}}$, taking the forward difference between both members of $\mathbf{v}=\mathcal{A}(\mathbf{v})$, we have

$$
\Delta v_{k}=\phi^{-1}\left(\frac{1}{k^{n-1}} G[v](k)\right) .
$$


This fact, together with (2.7), yields that

$$
\Delta v_{1}=0, \quad \Delta v_{N-1}=\phi^{-1}\left(\frac{1}{(N-1)^{n-1}} G[v](N-1)\right)=0
$$

Accordingly, for all $k \in[2, N-1]_{\mathbb{Z}}$,

$$
\nabla\left(k^{n-1} \phi\left(\Delta v_{k}\right)\right)=n k^{n-1}\left[-\frac{g^{\prime}\left(\psi^{-1}\left(v_{k}\right)\right)}{\sqrt{1-\left(\Delta v_{k}\right)^{2}}}+g\left(\psi^{-1}\left(v_{k}\right)\right) H\left(\psi^{-1}\left(v_{k}\right), k\right)\right] .
$$

Therefore, we conclude that $\mathbf{v}$ is also a solution of (1.2). We easily get the converse.

To study problem (1.2) by the Brouwer degree, we consider the following homotopy:

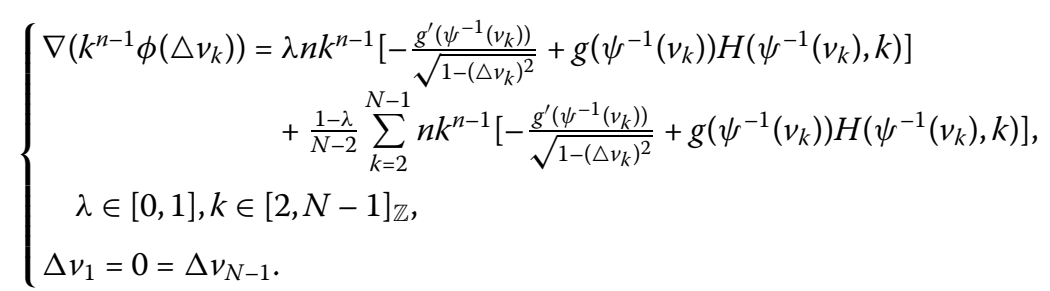

Notice that if $\lambda=1$, then (2.8) is problem (1.1). If $\lambda=0$, then (2.8) is the following problem:

$$
\left\{\begin{array}{l}
\nabla\left(k^{n-1} \phi\left(\Delta v_{k}\right)\right)=\frac{1}{N-2} \sum_{k=2}^{N-1} n k^{n-1}\left[-\frac{g^{\prime}\left(\psi^{-1}\left(v_{k}\right)\right)}{\sqrt{1-\left(\Delta v_{k}\right)^{2}}}+g\left(\psi^{-1}\left(v_{k}\right)\right) H\left(\psi^{-1}\left(v_{k}\right), k\right)\right], \\
k \in[2, N-1]_{\mathbb{Z}}, \\
\Delta v_{1}=0=\Delta v_{N-1} .
\end{array}\right.
$$

Equivalently, $v_{k}=c(c \in \mathbb{R})$ is a solution of the equation

$$
\frac{1}{N-2} \sum_{k=2}^{N-1} n k^{n-1}\left[-g^{\prime}\left(\psi^{-1}\left(v_{k}\right)\right)+g\left(\psi^{-1}\left(v_{k}\right)\right) H\left(\psi^{-1}\left(v_{k}\right), k\right)\right]=0 .
$$

For $\lambda \in(0,1]$, it follows from Proposition 2.1 that

$$
\sum_{k=2}^{N-1} n k^{n-1}\left[-\frac{g^{\prime}\left(\psi^{-1}\left(v_{k}\right)\right)}{\sqrt{1-\left(\triangle v_{k}\right)^{2}}}+g\left(\psi^{-1}\left(v_{k}\right)\right) H\left(\psi^{-1}\left(v_{k}\right), k\right)\right]=0
$$

Therefore (2.8) becomes

$$
\left\{\begin{array}{l}
\nabla\left(k^{n-1} \phi\left(\Delta v_{k}\right)\right)=\lambda n k^{n-1}\left[-\frac{g^{\prime}\left(\psi^{-1}\left(v_{k}\right)\right)}{\sqrt{1-\left(\Delta v_{k}\right)^{2}}}+g\left(\psi^{-1}\left(v_{k}\right)\right) H\left(\psi^{-1}\left(v_{k}\right), k\right)\right] \\
k \in[2, N-1]_{\mathbb{Z}} \\
\Delta v_{1}=0=\Delta v_{N-1}
\end{array}\right.
$$

A similar argument shows that, conversely, (2.9) implies (2.8), so that they are equivalent for $\lambda \in(0,1]$. 
Let $\|\Delta \mathbf{v}\|_{\infty}:=\max _{k \in[2, N-1]_{\mathbb{Z}}}\left|\Delta v_{k}\right|$, and let $\Gamma<1$ be a constant. Consider the operators $\mathcal{A}:\left\{\mathbf{v} \in W^{N-2}:\|\Delta \mathbf{v}\|_{\infty} \leq \Gamma\right\} \times[0,1] \rightarrow W^{N-2}$ given by $\mathcal{A}(\mathbf{v}, \lambda)=\mathbf{y}^{[\lambda]}$, that is,

$$
y_{k}^{[\lambda]}=v_{2}+\frac{1}{N-2} G[v](N-1)+\sum_{j=2}^{k-1} \phi^{-1}\left(\frac{\lambda}{j^{n-1}} G[v](j)\right), \quad k \in[2, N-1]_{\mathbb{Z}} .
$$

It is easy to check that $\mathcal{A}(\cdot, \lambda)$ is a compact operator.

Lemma 2.1 For $\lambda \in[0,1], \mathbf{v} \in W^{N-2}$ satisfies $\mathbf{v}=\mathcal{A}(\mathbf{v}, \lambda)$ if and only if $\mathbf{v}$ is a solution of (2.9).

Proof We can deduce Lemma 2.1 by similar arguments as in the proof of Proposition 2.2,

Lemma 2.2 Let (A1), (A2), (1.4), and (1.5) hold. Then there exist two constants $\delta_{*}$ and $\delta^{*}$ satisfying $\delta_{*}<0<\delta^{*}$ such that, for any solution $(\lambda, \mathbf{v})$ of $(2.9)$, we have

$$
\delta_{*}-2(N-3)<\|\mathbf{v}\|_{\infty}<\delta^{*}+2(N-3)
$$

Proof By Lemma $2.1,(\lambda, \mathbf{v})$ is a solution of (2.9) for some $\lambda \in[0,1]$ if and only if $\mathbf{v}=\mathcal{A}(\mathbf{v}, \lambda)$. By a simple calculation we have

$$
\Delta v_{k}=\phi^{-1}\left(\frac{\lambda}{k^{n-1}} G[v](k)\right), \quad k \in[2, N-1]_{\mathbb{Z}} .
$$

Since $\phi^{-1}: \mathbb{R} \rightarrow(-1,1)$, we can deduce that $\|\Delta \mathbf{v}\|_{\infty}<1$. Subsequently, letting $\widetilde{v}_{k}=v_{k}-v_{2}$, we have

$$
\left|\widetilde{v}_{k}\right|=\left|\sum_{i=2}^{k-1} \Delta v_{i}\right| \leq(k-2)\|\Delta \mathbf{v}\|_{\infty}<N-3
$$

and, accordingly,

$$
v_{2}-(N-3) \leq v_{k}=\widetilde{v}_{k}+v_{2} \leq v_{2}+(N-3)
$$

for all $k \in[2, N-1]_{\mathbb{Z}}$.

Note that (1.4) yields that $\psi: I \rightarrow \mathbb{R}$ is an increasing diffeomorphism, and hence $\psi^{-1}$ : $\mathbb{R} \rightarrow I$ is an increasing homeomorphism such that

$$
\lim _{s \rightarrow-\infty} \psi^{-1}(s)=\theta, \quad \lim _{s \rightarrow+\infty} \psi^{-1}(s)=\eta .
$$

It follows from (A2) that

$$
\lim _{v_{k} \rightarrow-\infty} \frac{g^{\prime}\left(\psi^{-1}\left(v_{k}\right)\right)}{g\left(\psi^{-1}\left(v_{k}\right)\right)}=+\infty, \quad \lim _{v_{k} \rightarrow+\infty} \frac{g^{\prime}\left(\psi^{-1}\left(v_{k}\right)\right)}{g\left(\psi^{-1}\left(v_{k}\right)\right)}=-\infty
$$

From this we get that there exists $\delta^{*}>0$ such that if $v_{k}>\delta^{*}$, then

$$
\frac{g^{\prime}\left(\psi^{-1}\left(v_{k}\right)\right)}{g\left(\psi^{-1}\left(v_{k}\right)\right)}<\min \{\underline{H}, 0\}
$$


where

$$
\underline{H}=\inf _{v \in I, k \in[2, N-1]_{\mathbb{Z}}} H(v, k) .
$$

Analogously, there exists $\delta_{*}<0$ such that

$$
\frac{g^{\prime}\left(\psi^{-1}\left(v_{k}\right)\right)}{g\left(\psi^{-1}\left(v_{k}\right)\right)}>\max \{\bar{H}, 0\}
$$

for all $v_{k} \in \mathbb{R}$ such that $v_{k}<\delta_{*}$, where

$$
\bar{H}=\sup _{v \in I, k \in[2, N-1]_{\mathbb{Z}}} H(v, k) .
$$

On the other hand, if $\lambda \in[0,1]$ and $\mathbf{v}=\mathcal{A}(\mathbf{v}, \lambda)$, then (2.7) holds, that is,

$$
G[v](N-1)=0 .
$$

We want to prove that $\delta_{*}-(N-3)<v_{2}<\delta^{*}+(N-3)$. If on the contrary we assume that $v_{2} \geq \delta^{*}+(N-3)$, then it follows from (2.12) that $v_{k} \geq \delta^{*}$ for all $k \in[2, N-1]_{\mathbb{Z}}$, and, using (2.14), we obtain

$$
G[v](N-1)>\sum_{k=2}^{N-1} n k^{n-1} g\left(\psi^{-1}\left(v_{k}\right)\right)\left[\frac{-g^{\prime}\left(\psi^{-1}\left(v_{k}\right)\right)}{g\left(\psi^{-1}\left(v_{k}\right)\right)}+\underline{H}\right]>0,
$$

which contradicts (2.7). Assume that $v_{2} \leq \delta_{*}-(N-3)$ and using (2.15), we can obtain a similar contradiction. Hence, $\delta_{*}-(N-3)<v_{2}<\delta^{*}+(N-3)$, and by $(2.12)$ we complete the proof.

Lemma 2.3 Let (A1), (A2), (1.4), and (1.5) hold. Then there exists a constant $\gamma^{*}<1$ such that, for any solution $(\lambda, \mathbf{v})$ of $(2.8)$, we have

$$
\|\Delta \mathbf{v}\|_{\infty} \leq \gamma^{*}
$$

Proof It is obvious that the result is true for $\lambda=0$. On the other hand, for any $\lambda \in[0,1]$, every solution $\mathbf{v}$ of (2.8) satisfies (2.9), and therefore, summing both members of (2.9) from 2 to $k$, together with the boundary conditions, we have

$$
k^{n-1} \phi\left(\Delta v_{k}\right)=\lambda G[v](k),
$$

where $G[v](k)$ is given by (2.5). Let us define

$$
C=\max \left\{g(v)|H(v, k)|: k \in[2, N-1]_{\mathbb{Z}}, v \in \widehat{I}\right\} .
$$


If $\left|\Delta v_{\rho}\right|=\max _{k \in[2, N-1]_{\mathbb{Z}}}\left|\Delta v_{k}\right|=\gamma<1$, then it follows from (2.16) that

$$
\begin{aligned}
\rho^{n-1} \frac{\left|\triangle v_{\rho}\right|}{\sqrt{1-\left|\triangle v_{\rho}\right|^{2}}} & \leq \sum_{i=2}^{\rho} n i^{n-1}\left[\frac{\left|g^{\prime}\left(\psi^{-1}\left(v_{i}\right)\right)\right|}{\sqrt{1-\left|\Delta v_{i}\right|^{2}}}+g\left(\psi^{-1}\left(v_{i}\right)\right)\left|H\left(\psi^{-1}\left(v_{i}\right), i\right)\right|\right] \\
& \leq \sum_{i=2}^{\rho} n i^{n-1}\left[\frac{\beta}{\sqrt{1-\left|\Delta v_{\rho}\right|^{2}}}+C\right] \\
& \leq\left[\frac{\beta}{\sqrt{1-\left|\triangle v_{\rho}\right|^{2}}}+C\right] \rho^{n} .
\end{aligned}
$$

Since $\rho \in[2, N-1]_{\mathbb{Z}}$, we have

$$
\gamma \leq\left[\beta+C \sqrt{1-\gamma^{2}}\right](N-1) .
$$

Let $f(\gamma)=\gamma-\left[\beta+C \sqrt{1-\gamma^{2}}\right](N-1)$. Recalling that $N<\frac{1}{\beta}+1$, we have

$$
f(0)=-(\beta+C)(N-1)<0, \quad f(1)=1-\beta(N-1)>0,
$$

and, accordingly, (2.17) is solvable, that is, we can get a fixed $\gamma^{*}<1$ with $\gamma<\gamma^{*}$.

\section{The proof of main results}

Proof of Theorem 1.1 Let

$$
\Omega=\left\{\mathbf{v} \in W^{N-2}:\|\Delta \mathbf{v}\|_{\infty}<\gamma^{*}, \delta_{*}-2(N-3)<\|\mathbf{v}\|_{\infty}<\delta^{*}+2(N-3)\right\},
$$

and let $\mathcal{A}$ be the fixed point operator defined in Lemma 2.1 .

By Lemma 2.2, Lemma 2.3, and the homotopy invariance of the Brouwer degree, we get that

$$
d_{B}[I-\mathcal{A}(\cdot, 0), \Omega, 0]=d_{B}[I-\mathcal{A}(\cdot, 1), \Omega, 0] .
$$

At the same time, by the reduction property of the Brouwer degree we know that

$$
\begin{aligned}
d_{B}[I-\mathcal{A}(\cdot, 0), \Omega, 0] & = \pm d_{B}[\kappa,(-\rho-2(N-3), \rho+2(N-3)), 0] \\
& = \pm \frac{\operatorname{sign}(\kappa(\rho+2(N-3)))-\operatorname{sign}(\kappa(-\rho-2(N-3)))}{2} \\
& = \pm 1
\end{aligned}
$$

where $\kappa$ is a continuous function from $\mathbb{R}$ to $\mathbb{R}$ of the following form:

$$
\kappa(x)=\sum_{k=2}^{N-1} n k^{n-1}\left[-g^{\prime}\left(\psi^{-1}(x)\right)+g\left(\psi^{-1}(x)\right) H\left(\psi^{-1}(x), k\right)\right] .
$$

Therefore $d_{B}[I-\mathcal{A}(\cdot, 1), \Omega, 0]= \pm 1$. It follows from the existence property of the Brouwer degree that there exists $\mathbf{v} \in \Omega$ satisfying $\mathbf{v}=\mathcal{A}(\mathbf{v}, 1)$. By Lemma 2.1 it is a solution of (1.2). 
Proof of Theorem 1.2 We may obtain Theorem 1.2 applying the same method (with obvious changes) as in the proof of Theorem 1.1. However, because of omitting condition (1.4), the range of $\psi$ is $J=(\psi(\theta), \psi(\eta))$, and $\psi^{-1}: J \rightarrow I$ is no longer defined on $\mathbb{R}$. Therefore

$$
\lim _{v_{k} \rightarrow(\psi(\theta))^{+}} \psi^{-1}\left(v_{k}\right)=\theta, \quad \lim _{v_{k} \rightarrow(\psi(\eta))^{-}} \psi^{-1}\left(v_{k}\right)=\eta
$$

and by (A2), (2.13) is replaced by

$$
\lim _{v_{k} \rightarrow(\psi(\theta))^{+}} \frac{g^{\prime}\left(\psi^{-1}\left(v_{k}\right)\right)}{g\left(\psi^{-1}\left(v_{k}\right)\right)}=+\infty, \quad \lim _{v_{k} \rightarrow(\psi(\eta))^{-}} \frac{g^{\prime}\left(\psi^{-1}\left(v_{k}\right)\right)}{g\left(\psi^{-1}\left(v_{k}\right)\right)}=-\infty
$$

By (3.2) there exist $\delta_{*} \leq \delta^{*}$ in $(\psi(\theta), \psi(\eta))$ such that $v_{k} \in\left(\delta^{*}, \psi(\eta)\right)$ implies (2.14) and $v_{k} \in$ $\left(\psi(\theta), \delta_{*}\right)$ implies (2.15). Notice that if $v_{k} \in J$, then the operator $\mathcal{A}(\mathbf{v}, \lambda)$ is well-defined. Therefore, we must choose $N$ in Lemma 2.2 satisfying

$$
\psi(\theta)<\delta_{*}-2(N-3)<v_{k}<\delta^{*}+2(N-3)<\psi(\eta)
$$

for $k \in[2, N-1]_{\mathbb{Z}}$, that is,

$$
N<\tilde{N}_{H}:=\min \left\{\frac{1}{2}\left(\delta_{*}-\psi(\theta)\right)+3, \frac{1}{2}\left(\psi(\eta)-\delta^{*}\right)+3\right\} .
$$

On the other hand, to overcome the omitted condition (1.5), we define

$$
\begin{aligned}
M_{N}:=\max \left\{\left|g^{\prime}\left(\psi^{-1}\left(v_{k}\right)\right)\right|: v_{k} \in\left[\delta_{*}-2(N-3), \delta^{*}+2(N-3)\right]\right\}, \\
A_{N}=\max \left\{g\left(\psi^{-1}\left(v_{k}\right)\right)\left|H\left(\psi^{-1}\left(v_{k}\right), k\right)\right|: k \in[2, N-1]_{\mathbb{Z}},\right. \\
\left.\quad v_{k} \in\left[\delta_{*}-2(N-3), \delta^{*}+2(N-3)\right]\right\} .
\end{aligned}
$$

It is worth pointing out that $M_{N}$ and $A_{N}$ are well-defined since $N<\tilde{N}_{H}$. Note that they decrease as $N$ decreases. Similarly, if $\left|\Delta v_{\rho}\right|=\max _{k \in[2, N-1]_{\mathbb{Z}}}\left|\Delta v_{k}\right|=\gamma<1$, then

$$
\rho^{n-1} \frac{\left|\triangle v_{\rho}\right|}{\sqrt{1-\left|\Delta v_{\rho}\right|^{2}}} \leq\left[\frac{M_{N}}{\sqrt{1-\left|\Delta v_{\rho}\right|^{2}}}+A_{N}\right] \rho^{n}
$$

which yields that

$$
\gamma \leq\left[M_{N}+A_{N} \sqrt{1-\gamma^{2}}\right](N-1)
$$

Combining this with the fact that $M_{N}$ decreases as $N$ decreases, there clearly exists $\widehat{N}_{H}>0$ such that $M_{N}(N-1)<1$ for any $N<\widehat{N}_{H}$ solving (3.3), and accordingly, we can obtain a fixed $\gamma^{*}<1$ such that $\gamma<\gamma^{*}$.

Now, take $N_{H}=\min \left\{\tilde{N}_{H}, \widehat{N}_{H}\right\}$ and let $N<N_{H}$. Then $\mathcal{A}(\nu, \lambda)$ is well-defined on the set

$$
\Omega=\left\{\mathbf{v} \in W^{N-2}:\|\Delta \mathbf{v}\|_{\infty}<\gamma^{*}, \delta_{*}-2(N-3)<\|\mathbf{v}\|_{\infty}<\delta^{*}+2(N-3)\right\},
$$

and similarly to the proof of Theorem 1.1, we can obtain the desired result. 
Remark 3.1 Checking the proofs of Theorem 1.1 and 1.2, we find that with some obvious changes, a similar existence result can be established for the quasilinear periodic boundary value problem

$$
\left\{\begin{array}{l}
\nabla\left(k^{n-1} \phi\left(\Delta v_{k}\right)\right)=n k^{n-1}\left[-\frac{g^{\prime}\left(\psi^{-1}\left(v_{k}\right)\right)}{\sqrt{1-\left(\Delta v_{k}\right)^{2}}}+g\left(\psi^{-1}\left(v_{k}\right)\right) H\left(\psi^{-1}\left(v_{k}\right), k\right)\right], \quad k \in[1, N]_{\mathbb{Z}} \\
v_{0}=v_{N}, \quad v_{1}=v_{N+1} .
\end{array}\right.
$$

Example 3.1 Let us consider the discrete Neumann boundary value problem

$$
\left\{\begin{array}{l}
\nabla\left(k^{n-1} \frac{\Delta v_{k}}{\sqrt{1-\left(\Delta v_{k}\right)^{2}}}\right)=n k^{n-1}\left[\frac{\frac{1}{10} \sin \left(2 \psi^{-1}\left(v_{k}\right)\right)}{\sqrt{1-\left(\Delta v_{k}\right)^{2}}}+\frac{1}{10} \cos ^{2}\left(\psi^{-1}\left(v_{k}\right)\right) H\left(\psi^{-1}\left(v_{k}\right), k\right)\right], \\
k \in[2, N-1]_{\mathbb{Z}}, \\
\Delta v_{1}=0=\Delta v_{N-1} .
\end{array}\right.
$$

Obviously, $I=\left(-\frac{\pi}{2}, \frac{\pi}{2}\right)$, and $g:\left[-\frac{\pi}{2}, \frac{\pi}{2}\right] \rightarrow \mathbb{R}^{+}$is given by

$$
g(t)=\frac{1}{10} \cos ^{2} t
$$

Also, it is obvious that all assumptions of Theorem 1.1 are satisfied. In particular, $\frac{g^{\prime}(t)}{g(t)}=$ $-2 \tan t$ and $M=\frac{1}{10}$. Moreover, $N<11$ satisfies $N \in \mathbb{N}$ with $N \geq 4$.

Therefore, by Theorem 1.1 we get that (3.4) has at least one solution $\mathbf{v}$ for any $H$ : $\left[-\frac{\pi}{2}, \frac{\pi}{2}\right] \times[2, N-1]_{\mathbb{Z}} \rightarrow \mathbb{R}$.

Example 3.2 Let $I=(-1,1)$. The function $g:[-1,1] \rightarrow \mathbb{R}^{+}$can be given by

$$
t \mapsto \frac{1}{100}\left(1-t^{2}\right)
$$

for which $\frac{g^{\prime}(t)}{g(t)}=\frac{2 t}{t^{2}-1}, M=\frac{1}{50}$, and $N<51$.

\section{Acknowledgements}

We are very grateful to the anonymous referees for their valuable suggestions. Our research was supported by the NSFC (No. 11671322, No. 11361054, No. 11626188).

\section{Competing interests}

Both authors claim that they have no any competing interests.

\section{Authors' contributions}

The authors claim that the research was realized in collaboration with the same responsibility. Both authors read and approved the last version of the manuscript.

\section{Publisher's Note}

Springer Nature remains neutral with regard to jurisdictional claims in published maps and institutional affiliations.

Received: 17 September 2017 Accepted: 28 December 2017 Published online: 10 January 2018

\section{References}

1. Mawhin, J, Torres, PJ: Prescribed mean curvature graphs with Neumann boundary conditions in some FLRW spacetimes. J. Differ. Equ. 261, 7145-7156 (2016)

2. de la Fuente, D, Romero, A, Torres, PJ: Radial solutions of the Dirichlet problem for the prescribed mean curvature equation in a Robertson-Walker spacetime. Adv. Nonlinear Stud. 15, 171-181 (2015)

3. Agarwal, RP: On multipoint boundary value problems for discrete equations. J. Math. Anal. Appl. 96(2), 520-534 (1983) 
4. Bartnik, R, Simon, L: Spacelike hypersurfaces with prescribed boundary values and mean curvature. Commun. Math Phys. 87, 131-152 (1982-1983)

5. Bereanu, C, Jebelean, P, Torres, PJ: Multiple positive radial solutions for a Dirichlet problem involving the mean curvature operator in Minkowski space. J. Funct. Anal. 265(4), 644-659 (2013)

6. Bereanu, C, Mawhin, J: Existence and multiplicity results for some nonlinear problems with singular $\phi$-Laplacian. J. Differ. Equ. 243, 536-557 (2007)

7. Cheng, SY, Yau, ST: Maximal spacelike hypersurfaces in the Lorentz-Minkowski spaces. Ann. Math. 104, 407-419 (1976)

8. Coelho, I, Corsato, C, Obersnel, F, Omari, P: Positive solutions of the Dirichlet problem for the one-dimensional Minkowski-curvature equation. Adv. Nonlinear Stud. 12(3), 621-638 (2012)

9. $\mathrm{Ma}, \mathrm{R}, \mathrm{GaO}, \mathrm{H}, \mathrm{Lu}, \mathrm{Y}$ : Global structure of radial positive solutions for a prescribed mean curvature problem in a ball. J. Funct. Anal. 270(7), 2430-2455 (2016)

10. Ma, R, Lu, Y: Multiplicity of positive solutions for second order nonlinear Dirichlet problem with one-dimension Minkowski-curvature operator. Adv. Nonlinear Stud. 15, 789-803 (2015)

11. Treibergs, AE: Entire spacelike hypersurfaces of constant mean curvature in Minkowski space. Invent. Math. 66, 39-56 (1982)

12. Corsato, C, Obersnel, F, Omari, P: The Dirichlet problem for gradient dependent prescribed mean curvature equations in the Lorentz-Minkowski space. Georgian Math. J. 24(1), 113-134 (2017)

13. Corsato, C, Obersnel, F, Omari, P, Rivetti, S: Positive solutions of the Dirichlet problem for the prescribed mean curvature equation in Minkowski space. J. Math. Anal. Appl. 405, 227-239 (2013)

14. de la Fuente, D, Romero, A, Torres, PJ: Existence and extendibility of rotationally symmetric graphs with a prescribed higher mean curvature function in Euclidean and Minkowski spaces. J. Math. Anal. Appl. 446, 1046-1059 (2017)

15. Bereanu, C, Thompson, HB: Periodic solutions of second order nonlinear difference equations with discrete $\phi$-Laplacian. J. Math. Anal. Appl. 330, 1002-1015 (2007)

16. Bereanu, C, Mawhin, J: Boundary value problems for second-order nonlinear difference equations with discrete $\phi$-Laplacian and singular $\phi$. J. Differ. Equ. Appl. 14(10-11), 1099-1118 (2008)

17. $\mathrm{Ma}, \mathrm{R}, \mathrm{Lu}, \mathrm{Y}$ : Periodic solutions of second order nonlinear difference equations with singular $\phi$-Laplacian operator Discrete Dyn. Nat. Soc. 2014, Article ID 637242 (2014)

18. Chen, T, Ma, R: Solvability for some boundary value problems with discrete $\phi$-Laplacian operators. Adv. Differ. Equ. 2015, $139(2015)$

19. Wang, Z, Qian, L, Lu, S, Cao, J: Existence and uniqueness of periodic solutions for a kind of Duffing type equation with two deviating arguments. Nonlinear Anal. 73(9), 3034-3043 (2010)

20. Wang, Z, Lu, S, Cao, J: Existence of periodic solutions for a $p$-Laplacian neutral functional differential equation with multiple variable parameters. Nonlinear Analysis, Series A 72(2), 734-747 (2010)

21. Mawhin, J, Szymańska-Dębowska, K: Second-order ordinary differential systems with nonlocal Neumann conditions at resonance. Ann. Mat. Pura Appl. 195, 1605-1617 (2016)

22. Feltrin, G, Zanolin, F: Multiplicity of positive periodic solutions in the superlinear indefinite case via coincidence degree. J. Differ. Equ. 262(8), 4255-4291 (2017)

23. Boscaggin, A, Feltrin, G, Zanolin, F: Pairs of positive periodic solutions of nonlinear ODEs with indefinite weight: a topological degree approach for the super-sublinear case. Proc. R. Soc. Edinb. A 146(3), 449-474 (2016)

24. Gaines, R, Mawhin, J: Coincidence Degree and Nonlinear Differential Equations. Springer, Berlin (1977)

25. Mawhin, J: Boundary value problems at resonance for vector second-order nonlinear ordinary differential equations. In: Equadiff IV. Lecture Notes in Math., vol. 703, pp. 241-249. Springer, Berlin (1979)

26. Mashhoon, B, Partovi, MH: Uniqueness of the Friedmann-Lemaitre-Robertson-Walker universes. Phys. Rev. D 30(8), 1839-1842 (1984)

27. Pinto-Neto, N, Trajtenberg, PI: The Hamiltonian of asymptotically Friedmann-Lemaître-Robertson-Walker spacetimes. Gen. Relativ. Gravit. 36(8), 1871-1881 (2004)

28. Rodnianski, I, Speck, J: The nonlinear future stability of the FLRW family of solutions to the irrotational Euler-Einstein system with a positive cosmological constant. J. Eur. Math. Soc. 15(6), 2369-2462 (2013)

29. Stoeger, WR: Almost FLRW observational cosmologies. In: Theory and Observational Limits in Cosmology, pp. 275-305. Specola Vaticana, Vatican City (1987)

\section{Submit your manuscript to a SpringerOpen ${ }^{\circ}$ journal and benefit from:}

- Convenient online submission

- Rigorous peer review

- Open access: articles freely available online

- High visibility within the field

- Retaining the copyright to your article

Submit your next manuscript at $\boldsymbol{s p r i n g e r o p e n . c o m ~}$ 